\title{
Subgrid parameterisations for high resolution atmospheric flows
}

\author{
V. Kitsios ${ }^{1} \quad$ J. S. Frederiksen ${ }^{2} \quad$ M. J. Zidikheri ${ }^{3}$
}

(Received 21 December 2010; revised 6 June 2011)

\begin{abstract}
Numerical and computational methods are developed for the Large Eddy Simulation of atmospheric flows on a sphere with a spectral quasigeostrophic model. The subgrid scales of motion are parameterised using a net eddy viscosity that is derived from a high resolution reference Direct Numerical Simulation with 504 zonal and total wavenumbers or $1536 \times 768$ grid points (longitude by latitude). Simulations are undertaken for a wide range of truncation wavenumbers to determine the influence of resolution on the net eddy viscosity. A universal scaling law for these coefficients is established for application to large eddy simulations of more general geophysical flows.
\end{abstract}

\section{Contents}

\section{Introduction}

http://anziamj . austms.org.au/ojs/index.php/ANZIAMJ/article/view/3800 gives this article, (c) Austral. Mathematical Soc. 2011. Published June 29, 2011. ISSN 1446-8735. (Print two pages per sheet of paper.) Copies of this article must not be made otherwise available on the internet; instead link directly to this URL for this article. 
2 Two-level spectral quasigeostrophic equations

C273

3 Stochastic subgrid scale model

C275

4 Scaling of the net eddy viscosity

C277

5 Self consistency of the bare viscosity

C281

6 Concluding remarks

C284

References

C285

\section{Introduction}

High Reynolds number atmospheric flows cover a vast range of scales making it unfeasible to adequately resolve all scales of motion. In geophysical fluid dynamics one therefore resorts to Large Eddy Simulation (LES) in which the large eddies are resolved on a computational grid and the remaining small scales are represented by a subgrid parameterisation. The first such parameterisation was the ad hoc deterministic Smagorinsky eddy viscosity model [6], in which the subgrid scale dissipation was related to the local strain rate via a specified parameter. An ad hoc stochastic version of this model was subsequently proposed by Leith [5]. Frederiksen and Davies [3] studied barotropic atmospheric flows and used turbulence closure theory to develop self consistent eddy viscosity and stochastic backscatter parameterisations with no tuning parameters needed. Frederiksen and Kepert [4] subsequently developed an essentially equivalent stochastic modelling approach, in which the eddy viscosity and stochastic backscatter terms are determined from a reference Direct Numerical Simulation (DNS) data set, in an effort to widen the applicability of the closure. In this context, a DNS is understood to be a highly resolved simulation with a wavenumber dependent bare viscosity to account for the unresolved scales of motion. 
The aim of the present study is to search for universal scaling laws of the wavenumber dependent viscosity by employing the subgrid modelling method of Frederiksen and Kepert [4]. This would make the parameterisations more generally applicable and remove the need to determine them from a reference DNS. Section 2 summarises the quasigeostrophic potential vorticity equation (QGPVE) $[1,7]$ used in our simulations of a turbulent baroclinic atmospheric flow. Section 3 presents the LES version of the QGPVE, along with the details of the stochastic modelling of the subgrid scales. Various LESs are then undertaken in Section 4 to investigate how the coefficients of the subgrid scale model change with resolution; a universal scaling of these coefficients is proposed. In Section 5 assess the self consistency of the scaling with the bare viscosity in the DNS.

\section{Two-level spectral quasigeostrophic equations}

For our study of subgrid scale parameterisations we employ the two level quasigeostrophic model of Frederiksen [1]. The QGPVE is derived on the basis of hydrostatic and approximate geostrophic balance. Here, the vorticity is represented at two discrete vertical levels, with $j=1$ representing the upper level at $250 \mathrm{hPa}(z \approx 10 \mathrm{~km})$, and $j=2$ the lower level at $750 \mathrm{hPa}$ $(z \approx 2.5 \mathrm{~km})$. The system is non-dimensionalised by the radius of the Earth $(\mathrm{a}=6371 \mathrm{~km})$ as a length scale and the inverse of the Earth's angular velocity $\left(\Omega=7.292 \times 10^{-5} \mathrm{~s}^{-1}\right)$ as a time scale.

The QGPVE is spectrally discretised by expanding the field variables in spherical harmonics with zonal (longitudinal) wavenumber $m$ and the total wavenumber $n$. Note the latitudinal (meridional) wavenumber is $n-m$. The evolution equation for the reduced potential vorticity spectral coefficients $\left(\mathbf{q}_{\mathfrak{m} \mathfrak{j}}^{j}\right)$ is [1]

$$
\frac{\partial q_{m n}^{j}}{\partial t}=i \sum_{p, q} \sum_{r, s} \kappa_{n q s}^{m p r} \psi_{-p q}^{j} q_{-r s}^{j}-i \omega_{m n} \zeta_{m n}^{j}-\alpha^{j} \zeta_{m n}^{j}+\kappa_{n}\left(\tilde{q}_{m n}^{j}-q_{m n}^{j}\right)
$$




$$
-\sum_{l=1}^{2} D_{0}^{j l}(m, n) q_{m n}^{j}
$$

where

$$
\mathrm{q}_{\mathrm{mn}}^{\mathrm{j}}=\zeta_{\mathrm{mn}}^{\mathrm{j}}+(-1)^{\mathrm{j}} \mathrm{F}_{\mathrm{L}}\left(\psi_{\mathrm{mn}}^{1}-\psi_{\mathrm{mn}}^{2}\right) .
$$

Here, $\zeta_{m n}^{j}=-\mathfrak{n}(n+1) \psi_{m n}^{j}$ are the spectral coefficients of the vorticity and $\psi_{m n}^{j}$ the streamfunction coefficients at level $j$. Also, $F_{L}$ is a layer coupling parameter, which is inversely proportional to the potential temperature difference between the two levels, and related to the Rossby radius of deformation by $r_{\text {Ros }}=1 / \sqrt{2 F_{L}}$. In (1) the summations are over the triangular truncated wavenumber set

$$
\begin{array}{r}
\mathbf{T}=\{\mathrm{p}, \mathrm{q}, \mathrm{r}, \mathrm{s}|-\mathrm{T} \leqslant \mathrm{p} \leqslant \mathrm{T},| \mathrm{p} \mid \leqslant \mathrm{q} \leqslant \mathrm{T}, \\
-\mathrm{T} \leqslant \mathrm{r} \leqslant \mathrm{T},|\mathrm{r}| \leqslant s \leqslant \mathrm{~T}\},
\end{array}
$$

with $T$ the DNs truncation wavenumber. The Rossby wave frequency $\omega_{\mathrm{m} n}=$ $-B m /(n(n+1))$, where $B=2$ with our non-dimensionalisation, and $K_{n q s}^{m p r}$ are the interaction coefficients detailed by Frederiksen and Kepert [4]. Linear drag is specified by $\alpha^{j}$ and the flow is relaxed towards $\tilde{\mathbf{q}}_{m \mathfrak{n}}^{j}$ with relaxation parameter $\kappa_{n}$.

In (1), the term $D_{0}^{j l}(m, n) q_{m n}^{j}$ represents the contribution of the unresolved scales of motion to the overall tendency. The linear operator, $D_{0}^{j l}(m, n)$, is written in general matrix anisotropic notation; however, in the present calculations it has the isotropic form

$$
D_{0}^{j l}(m, n)=v_{0}^{j l}(n) n(n+1),
$$

where $\mathfrak{n}(\mathfrak{n}+1)$ is the discrete form of the Laplacian operator. Also, the isotropic wavenumber dependent bare viscosity is

$$
v_{0}^{j l}(n)=\delta^{j l} v_{0}^{j j}(T)\left(\frac{n}{T}\right)^{\rho_{0}^{j}},
$$

where $\delta^{j l}$ is the Kronecker delta function, which ensures the off-diagonal elements of $\nu_{0}^{j l}(n)$ are zero. Here, $v_{0}^{j j}(T)$ is the value of the diagonal elements 
at the truncation wavenumber and the power $\rho_{0}^{j}$ controls the steepness of $\gamma_{0}^{j j}(n)$. This means that $\boldsymbol{v}_{\mathbf{0}}$, with elements $\boldsymbol{v}_{0}^{j l}$, and hence $\mathbf{D}_{\mathbf{0}}$, with elements $\mathrm{D}_{0}^{\mathrm{jl}}$, are isotropic diagonal matrices.

\section{$3 \quad$ Stochastic subgrid scale model}

The LES has reduced resolution compared with the DNS. The LES wavenumbers are confined to the set

$$
\begin{array}{r}
\mathbf{R}=\left\{p, q, r, s\left|-T_{R} \leqslant p \leqslant T_{R},\right| p \mid \leqslant q \leqslant T_{R},\right. \\
\left.-T_{R} \leqslant r \leqslant T_{R},|r| \leqslant s \leqslant T_{R}\right\},
\end{array}
$$

where $T_{R}$ is the LES truncation wavenumber and $T_{R}<T$. The subgrid wavenumber set can then be defined as $\mathbf{S}=\mathbf{T}-\mathbf{R}$. A stochastic subgrid scale model is presented that can replicate the DNS statistics of the large scales whilst truncating the model back from wavenumber set $\mathbf{T}$ to $\mathbf{R}$.

We find that successful LESs result from subgrid parameterisations that are inhomogeneous in the vertical and homogeneous but anisotropic in the horizontal. This allows the subgrid model to be applied locally in wavenumber space with coupling only in the vertical direction. This is consistent with the Quasi-diagonal Direct Interaction Approximation closure of Frederiksen [2] and the subgrid modelling study of Zidikheri and Frederiksen [7].

To facilitate a discussion on the flow decomposition, for a given wavenumber pair we let $\mathbf{q}$ equal the transpose of $\left(q_{m n}^{1}, q_{m n}^{2}\right)$. In this vector notation

$$
\mathbf{q}_{t}(t)=\mathbf{q}_{t}^{\mathbf{R}}(\mathbf{t})+\mathbf{q}_{t}^{\mathbf{S}}(\mathbf{t}),
$$

where $\mathbf{q}_{\mathbf{t}}$ is the tendency (or time derivative) of $\mathbf{q}$. Also, $\mathbf{q}_{\mathbf{t}}^{\mathbf{R}}$ is the tendency of the resolved scales where all triad interactions involve wavenumbers less than $T_{R}$, and consequently no parameterisation is required. For the remaining subgrid tendency, $\mathbf{q}_{\mathbf{t}}^{\mathbf{S}}$, at least one wavenumber component involved in the triad 
interactions is greater than $T_{R}$. The subgrid tendency is further decomposed such that

$$
\mathbf{q}_{\mathrm{t}}^{\mathbf{S}}(\mathrm{t})=\overline{\mathbf{f}}+\widehat{\mathbf{q}}_{\mathrm{t}}^{\mathbf{S}}(\mathrm{t}) \text {, }
$$

where $\overline{\mathbf{f}} \equiv \overline{\mathbf{q}_{t}^{\mathbf{S}}}$ is the time averaged subgrid tendency, and $\widehat{\mathbf{q}}_{t}^{\mathbf{S}}$ is the fluctuating component. In the present study the values of $\overline{\mathbf{f}}$ are determined from the DNS, and $\widehat{\mathbf{q}}_{\mathbf{t}}^{\mathbf{S}}$ is modelled using the following approach.

The fluctuating component of the subgrid tendency is represented by

$$
\widehat{\mathbf{q}}_{t}^{\mathbf{S}}(\mathrm{t})=-\mathbf{D}_{\mathbf{d}} \widehat{\mathbf{q}}(\mathrm{t})+\widehat{\mathbf{f}}(\mathrm{t}),
$$

where $\mathbf{D}_{\mathbf{d}}$ is the subgrid drain dissipation matrix, $\widehat{\mathbf{q}}$ is the fluctuating component of $\mathbf{q}$, and $\widehat{\mathbf{f}}$ is a random forcing vector. As the present simulations have two vertical levels, $\mathbf{D}_{\mathbf{d}}$ is a time independent $2 \times 2$ matrix, and $\widehat{\mathbf{f}}$ is a time dependent two element column vector. $\mathbf{D}_{\mathbf{d}}$ is determined by post-multiplying both sides of (9) by $\widehat{\mathbf{q}}^{\dagger}\left(\mathbf{t}_{0}\right)$, integrating over the decorrelation period $\tau$, and ensemble averaging to remove the contribution of the stochastic backscatter term $\widehat{\mathbf{f}}$. Rearranging for $\mathbf{D}_{\mathbf{d}}$ produces

$$
\mathbf{D}_{\mathbf{d}}=-\left\langle\int_{t_{0}}^{t} \widehat{\mathbf{q}}_{t}^{\mathbf{S}}(\sigma) \widehat{\mathbf{q}}^{\dagger}\left(t_{0}\right) d \sigma\right\rangle\left\langle\int_{t_{0}}^{t} \widehat{\mathbf{q}}(\sigma) \widehat{\mathbf{q}}^{\dagger}\left(t_{0}\right) d \sigma\right\rangle^{-1},
$$

where $\sigma$ is an integration variable, and $\dagger$ denotes the Hermitian conjugate for vectors and matrices. The angled brackets denote ensemble averaging, with each ensemble member determined by shifting the initial time $t_{0}$ and the final time $t=t_{0}+\tau$ forward by one time step. $\tau$ is chosen to capture the average subgrid contribution to the resolved scales.

The model for $\widehat{\mathbf{f}}$ is determined by calculating the non-linear noise covariance matrix given by $\mathcal{F}_{\mathrm{b}}=\mathbf{F}_{\mathrm{b}}+\mathbf{F}_{\mathrm{b}}^{\dagger}$, where $\mathbf{F}_{\mathrm{b}}=\left\langle\widehat{\mathbf{f}}(\mathrm{t}) \widehat{\mathbf{q}}^{\dagger}(\mathrm{t})\right\rangle$. By again postmultiplying both sides of (9) by $\widehat{\mathbf{q}}^{\dagger}\left(\mathbf{t}_{0}\right)$, and adding the conjugate transpose of (9) pre-multipled by $\widehat{\mathbf{q}}\left(\mathbf{t}_{0}\right)$, we obtain the Lyapunov or balance equation

$$
\left\langle\widehat{\mathbf{q}}_{\mathrm{t}}^{\mathbf{S}}(\mathrm{t}) \widehat{\mathbf{q}}^{\dagger}(\mathrm{t})\right\rangle+\left\langle\widehat{\mathbf{q}}(\mathrm{t}) \widehat{\mathbf{q}}_{\mathrm{t}}^{\mathbf{S}}(\mathrm{t})\right\rangle=-\mathbf{D}_{\mathbf{d}}\left\langle\widehat{\mathbf{q}}(\mathrm{t}) \widehat{\mathbf{q}}^{\dagger}(\mathrm{t})\right\rangle-\left\langle\widehat{\mathbf{q}}(\mathrm{t}) \widehat{\mathbf{q}}^{\dagger}(\mathrm{t})\right\rangle \mathbf{D}_{\mathbf{d}}^{\dagger}+\mathcal{F}_{\mathbf{b}},
$$


and $\mathcal{F}_{\mathbf{b}}$ is determined given that $\mathbf{D}_{\mathbf{d}}$ has been previously calculated. In general, the noise term $\widehat{\mathbf{f}}$ may be coloured, but we find that once $\mathcal{F}_{\mathbf{b}}$ has been calculated from (11), it is sufficient to model $\widehat{\mathbf{f}}$ as the white noise process $\left\langle\widehat{\mathbf{f}}(\mathrm{t}) \widehat{\mathbf{f}}^{\dagger}\left(\mathrm{t}^{\prime}\right)\right\rangle=\mathcal{F}_{\mathrm{b}} \delta\left(\mathrm{t}-\mathrm{t}^{\prime}\right)$. An eigenvalue decomposition of $\mathcal{F}_{\mathrm{b}}$ then produces a stochastic model for $\widehat{\mathbf{f}}$.

However, we adopt a deterministic expression for the backscatter. We define the backscatter and net linear operators by

$$
\begin{aligned}
& \mathbf{D}_{\mathbf{b}}=-\mathbf{F}_{\mathrm{b}}\left\langle\widehat{\mathbf{q}}(\mathrm{t}) \widehat{\mathbf{q}}^{\dagger}(\mathrm{t})\right\rangle^{-1}, \\
& \mathbf{D}_{\mathbf{n}}=\mathbf{D}_{\mathbf{d}}+\mathbf{D}_{\mathbf{b}},
\end{aligned}
$$

respectively. The subgrid tendency is then modelled by $\widehat{\mathbf{q}}_{t}^{\mathbf{S}}(t)=-\mathbf{D}_{\mathbf{n}} \widehat{\mathbf{q}}(\mathrm{t})$. The equation solved for the anisotropic LES is

$$
\begin{aligned}
\frac{\partial q_{m n}^{j}}{\partial t}= & i \sum_{p, q} \sum_{r, s} \kappa_{n q s}^{m p r} \psi_{-p q}^{j} q_{-r s}^{j}-i \omega_{m n} \zeta_{m n}^{j}-\alpha^{j} \zeta_{m n}^{j}+\kappa_{n}\left(\tilde{q}_{m n}^{j}-q_{m n}^{j}\right) \\
& -\sum_{l=1}^{2} D_{0}^{j l}(m, n) q_{m n}^{j}-\sum_{l=1}^{2} D_{n}^{j l}(m, n) \widehat{\mathfrak{q}}_{m n}^{l}+\bar{f}_{m n}^{j}
\end{aligned}
$$

solved over the wavenumber set $\mathbf{R}$. In the anisotropic case each wavenumber pair has a unique $\mathbf{D}_{\mathbf{n}}$. For the isotropic LES, $\mathbf{D}_{\mathbf{n}}$ is averaged over the zonal wavenumbers $m$, so that it is only a function of the total wavenumbers $n$. The net eddy viscosity $\boldsymbol{v}_{\mathbf{n}}=\mathbf{D}_{\mathbf{n}} /(\mathbf{n}(\mathrm{n}+1))$.

\section{Scaling of the net eddy viscosity}

Various DNSs and LESs are undertaken to identify how $\boldsymbol{v}_{\mathbf{n}}$ changes with resolution. The highest resolution DNS presented herein has a truncation wavenumber of $\mathrm{T}=504$, and is denoted by $\mathrm{T} 504$. This is equivalent to 1536 longitudinal and 768 latitudinal grid points, or a grid point every 
0.234 degrees. Along the equator, the distance between grid points is approximately $26 \mathrm{~km}$. The time step size for this simulation is $\Delta t=112$ seconds. All simulations are driven toward $\tilde{\mathbf{q}}_{\mathrm{mn}}^{j}$, by the relaxation parameter $\kappa_{\mathrm{n}}=10^{-6} \mathrm{~s}^{-1}$ for $n>15$ and $\kappa_{n}=0$ otherwise. The climate $\tilde{\mathbf{q}}_{m n}^{j}$ consists of a large westerly jet in the mid latitudes of each hemisphere, with $\tilde{\mathbf{q}}_{m n}^{j}$ non-zero only for $m=0$ (that is, a purely zonal jet). Further details on the structure of $\tilde{q}_{m n}^{j}$ was provided by Zidikheri and Frederiksen [7]. To be representative of atmospheric flows, the layer coupling parameter in SI units is $\mathrm{F}_{\mathrm{L}}=2.5 \times 10^{-12} \mathrm{~m}^{-2}$, with $r_{\text {Ros }}=4.47 \times 10^{5} \mathrm{~m}$ and a wavelength of $2 \pi r_{\text {Ros }}=2.81 \times 10^{6} \mathrm{~m}$. The associated non-dimensionalised Rossby wavenumber is $k_{\text {Ros }}=a / r_{\text {Ros }} \approx 14$. All simulations presented within resolve the large scale Rossby waves as $k_{\text {Ros }}<T_{R}<T$.

The anisotropic net eddy viscosity coefficients are calculated from the DNS data using (13) with $\tau=24 \Delta t$. For the T504 data set truncated back to $T_{R}=63$, the real component of the anisotropic net eddy viscosity $v_{n}^{11}(m, n)$, is illustrated in Figure 1. The eddy viscosity component $v_{n}^{11}(m, n)$ generally increase with $\mathrm{n}$ and have only a weak dependence on $\mathrm{m}$, and hence are approximately isotropic. The component $v_{n}^{22}(m, n)$ has a similar form to $v_{n}^{11}(m, n)$, but typically $80 \%$ of its magnitude. The cross elements, $v_{n}^{12}(n)$ and $v_{n}^{21}(n)$, are negligible in comparison. In all cases, the mean subgrid tendency $\overline{\mathbf{f}}$ is also found to be negligible. Each of these observations reinforce the use of a diagonal isotropic bare viscosity in (5). For the remainder of the article, we concentrate on the properties of $v_{n}^{11}(m, n)$.

The self similarity of the net eddy viscosity coefficients is most clearly illustrated by the isotropised profiles. The eddy viscosity component $\nu_{n}^{11}(m, n)$ is isotropised with $v_{n}^{11}(n)$ shown in Figure 2(a), and exhibits a cusp like shape approaching $T_{R}$. The T504 DNS data set is also truncated back to $T_{R}=126$ and $T_{R}=252$. The resulting profiles of $v_{n}^{11}(n)$ are illustrated in Figure 2(a), along with the bare viscosity profile $v_{0}^{11}(n)$. From this figure it is clear that the maximum value of $v_{n}^{11}(n)$ decreases with $T_{R}$, with the bare viscosity $v_{0}^{11}(n)$ having the smallest maximum value. This makes sense because as additional scales of motion are resolved by the grid, there are fewer subgrid scales that must be accounted for by the parameterisation, and the level of eddy viscosity 


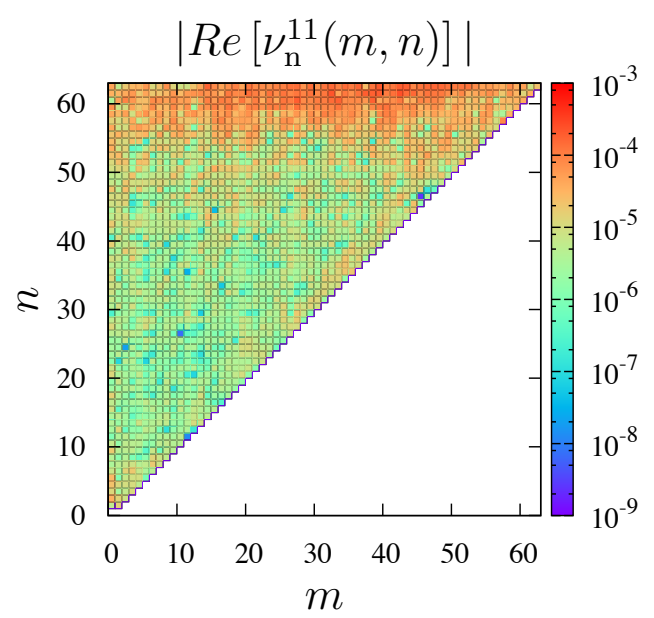

Figure 1: Net eddy viscosity subgrid coefficients $\left|\operatorname{Re}\left[v_{n}^{11}(m, n)\right]\right|$ required to truncate the T504 data set back to an LES data set with $T_{R}=63$.

should be reduced. Each of the four profiles is non-dimensionalised by its value at the truncation wavenumber denoted by $v_{n}^{11}\left(T_{R}\right)$ (or $v_{0}^{11}(T)$ in the case of the bare viscosity) and plotted in Figure 2(b) against $n / T_{R}$ (or $n / T$ for the bare viscosity). This figure accentuates that with this non-dimensionalisation, the steepness of the eddy viscosity profiles increases with the truncation wavenumber.

The changes in magnitude and slope of the net eddy viscosity profiles are quantified by fitting the curves to a power law function. Using a least squares approach, the $v_{n}^{11}(n)$ profiles are fit to the function

$$
v_{n}^{11}(n)=v_{n}^{11}\left(T_{R}\right)\left(\frac{n}{T_{R}}\right)^{\rho_{n}^{1}},
$$

where $v_{n}^{11}\left(T_{R}\right)$ is the value at truncation, and $\rho_{n}^{1}$ is the exponent. For the T504 DNS truncated back to $T_{R}=252,126$ and 63, the associated values of $\gamma_{n}^{11}\left(T_{R}\right)$ and $\rho_{n}^{1}$ are plotted as upward pointing triangles in Figure 3(a) and Figure 3(b) respectively. The component $v_{n}^{11}\left(T_{R}\right)$ is shown to decrease with $T_{R}$, and 


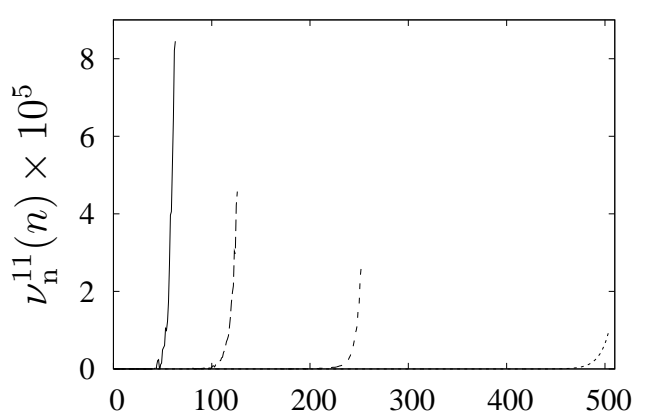

(a)

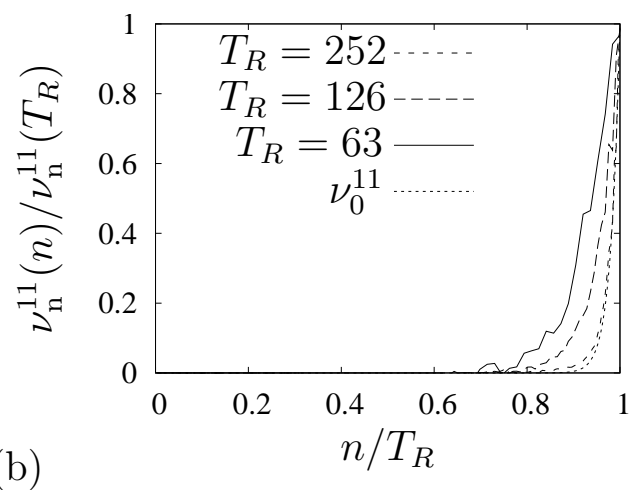

FiguRE 2: Isotropic net eddy viscosity subgrid coefficients derived from the T504 DNs data set: (a) $v_{n}^{11}(n)$; and (b) non-dimensionalised such that $v_{n}^{11}(n) / v_{n}^{11}\left(T_{R}\right)$ is plotted against $n / T_{R}$. The legend in (b) also represents the data in (a). The bare viscosity $\nu_{0}^{11}$ is also included in these figures.

$\rho_{\mathfrak{n}}^{1}$ increases with $T_{R}$. This means that as more scales are resolved the net eddy viscosity profiles are reducing in magnitude and becoming steeper, which is consistent with the results in Figure 2.

Additional DNS calculations are performed to confirm the functional dependence of $\nu_{n}^{11}\left(T_{R}\right)$ and $\rho_{n}^{1}$ on the LES truncation wavenumber $T_{R}$. The new DNSs are truncated at $T=252$ and $T=126$, with these data sets referred to as T252 and T126 respectively. The $\boldsymbol{v}_{\mathbf{0}}$ coefficients of the T252 data set are estimated from $\boldsymbol{v}_{\mathbf{n}}$ of T504 truncated back to $T_{R}=252$. Likewise the $\boldsymbol{v}_{\mathbf{0}}$ coefficients of the T126 data set are estimated from $\boldsymbol{v}_{\mathbf{n}}$ of T504 truncated back to $T_{R}=126$. The T252 DNS is in turn truncated back to $T_{R}=126,63$ and 31, with the associated values of $\nu_{n}^{11}\left(T_{R}\right)$, and $\rho_{n}^{1}$ plotted as downward pointing triangles in Figure 3. The T126 DNs is truncated back to several levels between $T_{R}=31$ and 63 , with the values of $\nu_{n}^{11}\left(T_{R}\right)$, and $\rho_{n}^{1}$ plotted as open circles. A least squares regression line is determined for the $v_{n}^{11}\left(T_{R}\right)$ data 


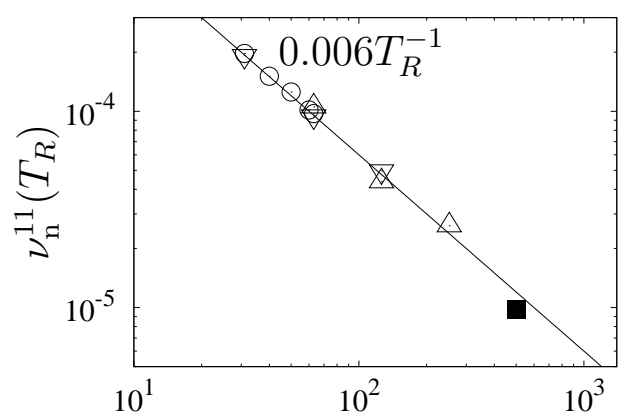

(a)

$T_{R}$

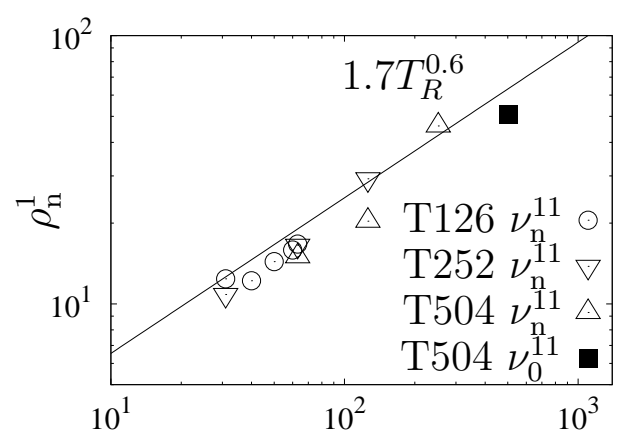

(b)

$T_{R}$

Figure 3: The scaling of the subgrid coefficients properties: (a) $\nu_{n}^{11}\left(T_{R}\right)$; and (b) $\rho_{n}^{1}$, determined from three DNS data sets T504, T252 and T126 truncated back to various values of $T_{R}$. The legend in (b) represents the data in (a).

points in Figure 3(a) producing the functional dependence

$$
v_{n}^{11}\left(T_{R}\right)=0.006 T_{R}^{-1}
$$

with a correlation coefficient of 0.997 . A least squares regression line is also calculated for the $\rho_{\mathfrak{n}}^{1}$ data points in Figure 3(b) producing the function

$$
\rho_{n}^{1}\left(T_{R}\right)=1.7 T_{R}^{0.6}
$$

with a correlation coefficient of 0.960 . The values of $\nu_{0}^{11}$ and $\rho_{0}^{1}$ for the T504 DNS are also plotted on Figure 3(a) and Figure 3(b) respectively as solid squares, and follow the trend line.

\section{Self consistency of the bare viscosity}

Just as the bare viscosity $\boldsymbol{v}_{\mathbf{0}}$ represents the contribution of the unresolved scales in the DNS to the tendency over the wavenumber set $\mathbf{T}$, the net eddy 


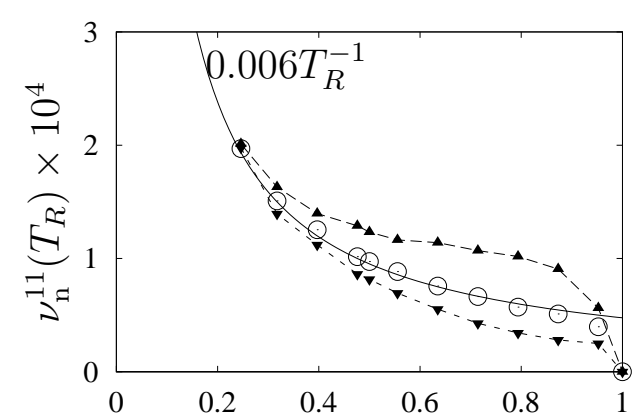

(a)

$$
T_{R} / T
$$

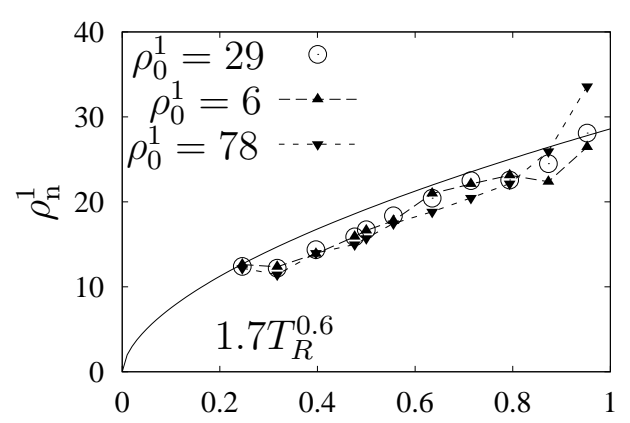

(b)

$$
T_{R} / T
$$

Figure 4: Using the T126 DNS data set, the sensitivity to $\rho_{0}^{1}$ of the subgrid coefficients properties: (a) $\nu_{n}^{11}\left(T_{R}\right)$; and (b) $\rho_{n}^{1}$, for various values of $T_{R}$.

viscosity $\boldsymbol{v}_{\mathbf{n}}$ represents the contribution of the subgrid scales $\mathbf{S}$ to the tendency of the LES resolved scales $\mathbf{R}$. Therefore, we propose that the scaling observed for $\boldsymbol{v}_{\mathbf{n}}$, is also applicable to $\boldsymbol{v}_{\mathbf{0}}$. It was shown previously in Figure 3 that $\boldsymbol{v}_{\mathrm{n}}$ and $\boldsymbol{v}_{\mathbf{0}}$ have consistent scaling. The sensitivity of this result is assessed below. As an additional check, DNSs are run with $\boldsymbol{v}_{\mathbf{0}}$ profiles generated using the scaling functions. The resulting spectrum are compared to the spectrum from the highest resolution T504 case.

Thus far the scaling laws for $\boldsymbol{v}_{\mathbf{n}}$ have been identified by running a series of DNSs with a prescribed $\boldsymbol{v}_{\mathbf{0}}$ truncated at various $T_{R}$. The sensitivity of the results is now determined by running additional DNSs with varying $\boldsymbol{v}_{\mathbf{0}}$. Specifically the T126 DNS is run with three different bare viscosity profiles, each of which are truncated back to several levels between $T_{R}=31$ and 126. In each case $v_{0}$ is calculated according to (5), with $v_{0}^{\mathrm{jj}}\left(T_{R}\right)$ fixed and equal to $\nu_{n}^{\mathrm{jj}}\left(T_{R}\right)$ of the T504 DNS truncated back to $T_{R}=126$. Three different values of $\rho_{0}^{j}$ are used: $\rho_{0}^{j}=29$, consistent with the previous T126 DNS; $\rho_{0}^{j}=6$, a typical value used in atmospheric DNS studies; and $\rho_{0}^{j}=78$, chosen to illustrate the impact of an excessively large value. The impact of the $\boldsymbol{v}_{0}$ profiles on $v_{n}^{11}\left(T_{R}\right)$ at various $T_{R}$ is first assessed. The trend line from Figure 3(a) is included in 
Figure 4(a), but this time plotted on a linear scale with $T_{R} / T$ the independent variable. The DNS calculations are repeated with $\rho_{0}^{j}=29,6$ and 78, and the resulting values of $v_{n}^{11}\left(T_{R}\right)$ added to Figure 4(a). The values of $v_{n}^{11}\left(T_{R}\right)$ appear to deviate from each other for $T_{R} / T>0.5$. The same is true regarding the agreement of $\rho_{n}^{1}$ determined from the various DNSs in Figure 4(b). In general, the closer $T_{R}$ is to $T$ the greater influence $\boldsymbol{v}_{\mathbf{0}}$ has on $\boldsymbol{v}_{\mathbf{n}}$. However, in close proximity to $T, v_{n}^{11}\left(T_{R}\right)$ approaches zero regardless of $\boldsymbol{v}_{\mathbf{0}}$. This is because there are no subgrid scales at $T_{R}=T$, and by definition $\boldsymbol{v}_{\mathbf{n}}=0$. To ensure the limiting case at $T=T_{R}$ and the prescribed $\boldsymbol{v}_{0}$ had minimal impact on the scaling functions describing $\boldsymbol{v}_{\mathbf{n}}$, the regression lines in Figure 3 were calculated using only the data points with $T_{R} / T \leqslant 0.5$.

We now verify that a lower resolution DNS with $\boldsymbol{v}_{\mathbf{0}}$ defined using the scaling laws can replicate the statistics of a higher resolution DNS. A comparison is made between the time and $m$ averaged kinetic energy spectrum on level one $\left(E_{K}^{1}\right)$ resulting from the T504, T252 and T126 DNss. The $\boldsymbol{v}_{\mathbf{0}}$ profiles for the T252 and T126 DNS are determined using the scaling functions (16) and (17) in conjunction with (5). Figure 5(a) illustrates good agreement between the $E_{K}^{1}$ from each of the DNSs. Importantly the energies in the low wavenumber large scales of motion agree. In the higher wavenumbers, the lower resolution cases decay slightly faster than the T504 case. The implications of using the incorrect scaling for $\boldsymbol{v}_{\mathbf{0}}$ is illustrated with application to the T126 DNS. Figure 5(b) illustrates $E_{k}^{1}$ resulting from the T126 DNSs with $\rho_{0}^{j}=29,6$ and 78. The spectrum labelled $\rho_{0}^{j}=29$ is the same as the T126 DNS spectrum in Figure 5(a). The spectrum labelled $\rho_{0}^{j}=78$ produces a turn up at the end of the spectrum due to the net eddy viscosity dropping off more rapidly. The spectrum from the $\rho_{0}^{j}=6$ case rapidly decays at the higher wavenumbers, with a turn up at the tail of the spectra. If the incorrect $\boldsymbol{v}_{\mathbf{0}}$ is applied, the tails of the energy spectrum are not correctly represented; however, at these resolutions the large scales still seem to be adequately captured. 


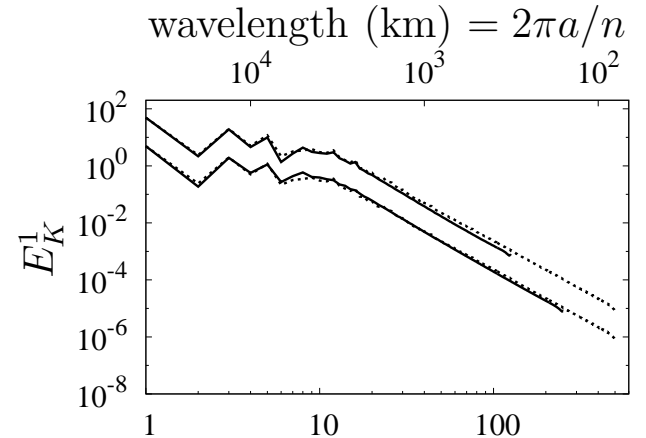

(a)

$n$ (b)

wavelength $(\mathrm{km})=2 \pi a / n$

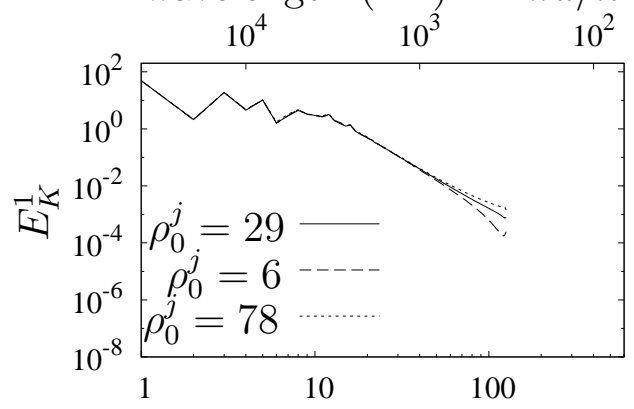

$n$

Figure 5: Comparison of $\mathrm{E}_{\mathrm{K}}^{1}$ between (a) the T504 DNS and the T126 DNS (top spectrum) and with the T252 DNs (bottom spectrum), and (b) the T126 DNs using three different values of $\rho_{0}^{j}$.

\section{Concluding remarks}

Data from a two level quasigeostrophic atmospheric model has been generated using DNS. Subgrid parameterisations consisting of a net eddy viscosity were generated from the DNS data at various truncation levels. Self similar scaling laws for the net eddy viscosity were determined from the results. At present the scaling laws are level dependent; however, recent work identified that these scalings are unified by using a level dependent enstrophy flux. These scaling laws may remove the need to produce high resolution reference DNS data sets, which would widen the applicability of the LES method to more general cases.

Acknowledgements V. Kitsios thanks the CSIRO Office of the Chief Executive for funding his post-doctoral position. 


\section{References}

[1] J. S. Frederiksen. Precursors to blocking anomalies: the tangent linear and inverse problems. J. Atmos. Sci., 55:2419-2436, 1998. doi:10.1175/1520-0469(1998)055¡2419:PTBATT¿2.0.CO;2 C273

[2] J. S. Frederiksen. Subgrid-scale parameterizations of eddy-topographic force, eddy viscosity and stochastic backscatter for flow over topography. J. Atmos. Sci, 56:1481-1493, 1999. doi:10.1175/1520-0469(1999)056¡1481:SSPOET¿2.0.CO;2 C275

[3] J. S. Frederiksen and A. G. Davies. Eddy viscosity and stochastic backscatter parameterizations on the sphere for atmospheric circulation models. J. Atmos. Sci, 54:2475-2492, 1997. doi:10.1175/1520-0469(1997)054¡2475:EVASBP ¿2.0.CO;2 C272

[4] J. S. Frederiksen and S. M. Kepert. Dynamical subgrid-scale parameterizations from Direct Numerical Simulations. J. Atmos. Sci., 63:3006-3019, 2006. doi:10.1175/JAS3795.1 C272, C273, C274

[5] C. E. Leith. Stochastic backscatter in a subgrid-scale model: Plane shear mixing layer. Phys. Fluids, 2(3):297-299, 1990. doi:10.1063/1.857779 C272

[6] J. Smagorinsky. General circulation experiments with the primitive equations: I. the basic experiment. Mon. Wea. Rev., 91(3):99-164, 1963. doi:10.1175/1520-0493(1963)091;0099:GCEWTP ¿2.3.CO;2 C272

[7] M. J. Zidikheri and J. S. Frederiksen. Stochastic subgrid parameterizations for simulations of atmospheric baroclinic flows. J. Atmos. Sci., 66:2844-2858, 2009. doi:10.1175/2009JAS3036 C273, C275, C278 


\section{Author addresses}

1. V. Kitsios, Centre for Australian Weather and Climate Research (CAWCR), CSIRO Marine and Atmospheric Research, Aspendale 3195, Australia.

2. J. S. Frederiksen, CAWCR, CSIRO Marine and Atmospheric Research, Aspendale 3195, Australia.

3. M. J. Zidikheri, CAWCR, Bureau of Meteorology, Melbourne, Victoria, Australia. 